\title{
Analysis on the Status Quo, Problems and Countermeasures of Zhengzhou Fresh Cold Chain Logistics Development
}

\author{
Xiang Li, Siqi Li \\ Administrative Engineering College, Zhengzhou University, No.100 Science Avenue, Zhengzhou, \\ China
}

Keywords: fresh cold chain logistics; status quo; development countermeasures.

Abstract: Cold chain logistics is closely related to people's lives. Zhengzhou has a superior geographic location and a large population density. It has a good cold chain logistics foundation. However, the development of Zhengzhou cold chain logistics is still not perfect. This paper describes the current development status of Zhengzhou fresh cold chain logistics. The existing problems and related countermeasures are proposed.

\section{Introduction}

Cold chain logistics generally refers to a systematic project in which the products are always in the specified temperature range during production, storage, transportation, sales, and before consumption to ensure product quality and reduce cargo damage (Qinggang Zhao,2011). At present, cold chain logistics is mainly used in the field of food fresh produce. In 2017, the transaction volume of China's fresh e-commerce market was about 139.13 billion yuan. The high-growth market demand made the development of fresh-chain cold chain logistics more rapid and more important.

\section{Research background and significance of FRESH cold chain logistics}

\subsection{The concept of fresh cold chain logistics}

Fresh cold chain logistics refers to the refrigerated fresh products in the production, transportation, storage, handling, circulation and processing, to the hands of consumers in all aspects of the low temperature environment has always been in order to ensure the quality of fresh products, reduce the loss of fresh products.

\subsection{The research background of fresh cold chain logistics}

According to the official website of Zhengzhou Municipal Bureau of Statistics, Zhengzhou City's total annual grain output was 1.532 million tons, cotton output was 2 million tons, oil production was 139,000 tons, vegetable output was 2.005 million tons, and total fruit output was 269,000 tons, the total output of meat is 236,000 tons, the number of eggs is 157,000 tons, the output of aquatic 
products is 138,000 tons, and the output of milk is 233,000 tons. The good agricultural foundation provides support for the development of fresh cold chain logistics.

Food is first in circulation. First of all, fresh products have the characteristics of seasonality, perishability, and difficulty in storage and transportation. Secondly, with the improvement of living standards, consumers are not only demanding more and more fresh foods from different localities. Moreover, it pays more attention to the green and safety of fresh products, but the standardization and automation of cold chain logistics in Zhengzhou City is low, which affects consumers' satisfaction with fresh products and fresh logistics, so the development of professional cold chain logistics has been become a top priority.

The background of the Belt and Road, smart logistics and cross-border fresh logistics provides new challenges and opportunities for Zhengzhou cold chain logistics. Therefore, the current situation of cold chain logistics in Zhengzhou should be improved in combination with the needs of consumers and the background of the times, so as to improve the service level of cold chain logistics in Zhengzhou.

\subsection{The research significance of fresh cold chain logistics}

Improving the service level of fresh cold chain logistics helps to ensure the quality of fresh products, promote the circulation of fresh products in the market, promote the development of agriculture, meet people's diversified demands for fresh agricultural products, and improve consumers' satisfaction and loyalty to fresh logistics.

\section{Development Status and Problems of Zhengzhou FRESH Cold Chain Logistics}

\subsection{Development Status of Fresh Cold Chain Logistics in Zhengzhou}

Zhengzhou's cold chain logistics has a good foundation for development. With the rapid development of Zhengzhou Fresh E-commerce market, the demand for vaccine products and biopharmaceuticals related to the cold chain is huge, and the scale and proportion of cold chain logistics is growing rapidly.It not only has the cold chain logistics companies such as Kaixue and Henan Xianyi, which can meet the domestic trunk cold chain logistics and urban cold chain logistics distribution, but also own the 5,000-square-meter Zhengzhou International Land Port, which can realize more than 10,000 kilometers of international cold chain transportation from Zhengzhou to Europe, which has been improved the international cold chain logistics service level in the context of the "Belt and Road".In addition, it is understood that the Zhongyuan Cold Chain Valley, located in Zhengzhou International Logistics Park, covers an area of 260,000 square meters, is expected to be put into operation in October this year. The strategic cooperation agreement to build China (Zhengzhou) cold chain trading center with an investment of 900 million yuan was also successfully signed on June 28 this year.

However, the development of Zhengzhou cold chain logistics is still not perfect. Not only does it lack professional cold chain transportation equipment, but the cost of cold chain logistics is still high. According to statistics, the annual demand for cold chain logistics services in Henan Province is about 36 million tons, the demand for cold chain cold storage has exceeded 20 million cubic meters, while the actual supply of Henan Province is only 6 million cubic meters, the per capita cold storage area is about 0.056 square meters, and in recent two years, Henan Province registered more than 5,000 standard cold chain vehicles, The total volume of the whole country is about 80 thousand. At present, the circulation rate of fruits and vegetables and meat products in Zhengzhou is less than $20 \%$, the rate of fresh food decay is more than $30 \%$, the transportation of cold chain logistics is mainly highway, and most of the last mile uses foam box and low temperature ice bag 
mode, and the cold chain circulation rate and refrigerated transport rate are still low.

\subsection{Problems of fresh cold chain logistics in Zhengzhou}

\subsubsection{The cold chain facilities and equipment behind}

The core of the cold chain is constant temperature, and the temperature needs to be controlled within a certain interval. Different reservoir areas also need to maintain different humidity. Generally speaking, the fresh-keeping warehouse and the frozen storage warehouse are not similar to the grooves under the refrigerated transport vehicle. The cold air circulation on the side close to the wall in the library is poor, and the temperature is the main factor affecting the quality of fresh products, such as leafy vegetables. Internal rot is caused by the heat generated by cellular respiration, but the temperature is too low, which will cause frostbite in the dish, and some of the dishes are not easy to be seen. If the potato is slightly frostbitten, the inside will turn black, but the appearance will not be seen, frostbite distributing food to consumers in the supply chain creates unnecessary troubles and troubles for consumers.

The cold chain transportation equipment is outdated, the low temperature in the cold chain transportation is difficult to reach, or the temperature is difficult to be constant after reaching. There are some agricultural product processing companies in the market that use non-cold chain vehicles to transport fresh products. For example, when transporting goods at close range, they use ordinary vans to transport products that require low-temperature storage and transportation. When arriving at the warehouse of a third-party logistics company, the temperature in the compartment is higher than the temperature required by the warehouse to receive the goods. In order to avoid re-transportation, they will insert the thermometer in the lowest temperature in the car to meet the specified temperature. If the temperature is not up to the requirement, it will be sent back to the company for refrigerating or freezing. Fresh products have undergone the process of freezing, thawing and refreezing during the whole process, which seriously affects the quality of the products. In addition, the cold chain logistics storage and transportation equipment connection needs to be improved, the refrigerated truck can not enter the cold storage, loading and unloading will cause a lot of cold air loss and equipment internal temperature fluctuations, the whole process cold chain can not be achieved (Xueguo Yuan, Ping Zou,2015).

This is especially true for the pharmaceutical cold chain. The temperature of each link is extremely important for the drug. The temperature change may make the drug reduce the efficacy or directly invalidate. However, the drug transportation process cannot be monitored in real time, and the loading and unloading is not eliminated. The quality of the car is not guaranteed by the influence of temperature and humidity inside the car.

\subsubsection{The degree of information is low}

Many cold chain logistics companies have emerged in the market, but most of them do not have a complete logistics information system. The low informationization makes the goods entering and leaving the warehouse, inventory, delivery and receiving process cumbersome and inefficient, so that the warehouse can not follow the principle of first-in, first-out, affecting the inventory turnover and the quality of fresh produce.

The imbalance between supply and demand caused by market information asymmetry is also a prominent problem. Zhengzhou agricultural product suppliers mostly appear in the form of retail investors. The bullwhip effect easily leads to the slow sales of retail fresh products. If it cannot be circulated in time, it can only let the fresh products rot and deteriorate, causing serious losses.

In addition, the lack of information in the supply chain leads to a large number of safe stocks, 
and increases the lead time of the order, which affects the flexibility and professional development of the entire supply chain.

\subsubsection{The cold chain logistics costs are high}

(1) High social costs and operating costs

The cold chain logistics is $40 \%-60 \%$ higher than the general logistics cost. First of all, cold chain logistics companies need a large area of land to build warehouses and because of the special requirements for temperature, the construction cost of cold storage is high, a standardized cold storage construction cost is equivalent to ordinary warehouse 4-5 times. Secondly, the cost of coldchain transport equipment is also higher than ordinary logistics transport equipment, it is reported that Zhengzhou International Land Port Company for international railway "cold-chain" transport of white refrigerated thermostatic containers, although similar to the shape of ordinary normal temperature containers, but the cost is more than 20 times the price of ordinary containers. Cold chain transport vehicle is more vulnerable to external temperature in the process of transportation, energy consumption is large, the stacking and handling of goods will affect the temperature of the car and its stability. Some companies have built their own cold-chain logistics to improve service quality and meet customer needs, increasing operating costs and ignoring the idleness and waste of resources brought about by seasonal products.

In addition, in order to ensure product quality and logistics timeliness, fresh e-commerce needs to expand off-line stores, but due to the lack of community channels, this not only raises operating costs, but also can not fundamentally solve the logistics problem.

(2) Lack of strong government supervision and policy support

Logistics is called "the third source of profit", and the development prospects of cold chain logistics are even broader. Due to the increase in people's demand, logistics enterprises have sprung up, but the logistics industry is mixed with small and medium-sized enterprises, lacking government supervision and clear industry norms. The small and medium-sized logistics enterprises that have been continuously joined have different implementation standards and the service level is also uneven. The price war is carried out for operating income, which has led to vicious competition in the industry.

The cold chain logistics industry lacks relevant policy support, transportation vehicles are not smooth, and energy consumption is high. The "first mile" and "last mile" are still pain points.

\section{Development Countermeasures of Zhengzhou FRESH Cold Chain Logistics}

\subsection{Strengthening the construction of fresh cold chain logistics facilities and equipment}

Innovative development of smart devices. Promote the application of advanced information technology and equipment, strengthen the innovation and research and development of cold chain logistics technology and facilities, build intelligent warehousing and logistics facilities, encourage logistics mechanization, automation equipment innovation research and development and promotion and application, improve logistics efficiency and warehousing modern management level, reduce logistics costs To accelerate the realization of the entire cold chain (Jianqi Zhang,2018).

Specifically, it can introduce automated three-dimensional library, store products with shelves, increase storage capacity utilization, innovate and develop warehouse automatic temperature control equipment, automatically adjust the temperature and humidity in the library, reduce the impact of manual activities; innovate and develop transportation equipment, upgrade refrigerated truck configuration. It can not only ensure the whole process of transportation at a suitable low temperature, but also reduce energy consumption and save logistics and transportation costs. 
Enhance the standardization of cold chain logistics equipment. The different standards of logistics equipment easily lead to duplicate work, which brings inconvenience to the transfer of work in different links of the whole cold chain. For fresh products, the standards of pallets in different links are inconsistent in the circulation process, resulting in inconvenience in the circulation of products at home and abroad, the inconvenience not only affects the timeliness but also increases the logistics cost.

\subsection{Improve the information level of fresh cold chain logistics}

\subsubsection{The introduction of advanced information systems}

In this era of information-driven, cold chain companies should rely on advanced information technologies such as the Internet, big data, and cloud computing to vigorously develop a new model of "Internet + cold chain logistics" and promote informationization and dataization of logistics activities.

Vendor Management Inventory (VMI) is a commonly used inventory management method for fresh cold chain. It can promote the integrated development of supply chain information, help enterprises reduce inventory costs, reduce order cycle, and reduce cargo damage and cargo gap.

Warehouse Management System (WMS) is a warehouse management software that standardizes and intelligently executes process management, enabling accurate and efficient integrated management of warehouses (Yafang Tian, Xu Feng, 2017). The Transportation Management Information System (TMS) is also essential in the supply chain. TMS is an information management system applied to enterprise logistics departments and third-party logistics organizations. It manages the business of order processing, transportation, distribution, carrier management, capacity management, return order management, receivable and payable management and returning management.

Cold chain logistics should rely on modern logistics equipment and logistics information management system, through the effective integration of upstream and downstream resources, optimize the operation process, shorten inventory and turnover time, reduce logistics costs, realize automation and informatization.

\subsubsection{Information integration}

Building intelligent cold chain logistics platform. For fresh products, the supply chain information should be maintained smoothly, eliminating the inconsistency between supply and demand caused by the imbalance of information, material sluggishness and other issues, and the source of production should be traceable. Cold chain logistics enterprises store information in the form of two-dimensional codes. Consumers can obtain the information of products in the whole cold chain circulation by sweeping codes, including storage conditions, temperature and humidity of vehicles in the process of transportation.

Cold chain logistics enterprises should regularly inspect and maintain equipment, record daily warehouse temperature and humidity, perform GPS positioning on each transportation equipment, check and record various indicators before departure, and set temperature and humidity change regulators in the vehicle. The temperature and humidity can be automatically adjusted according to the system settings, and the vehicle position and the temperature and humidity in the cabin can be automatically synchronized to the enterprise background supervision system to monitor the vehicles in transit. 


\subsection{Reduce the cost of fresh cold chain logistics}

\subsubsection{Multimodal transport}

The development of multimodal transport is an effective way to reduce the cost of logistics, and it is also the key work of comprehensive transport development during the 13th Five-Year Plan period. Zhengzhou is situated at the intersection of the land bridge passage and the Beijing-HarbinBeijing-Guangzhou passage in the state's "two horizontal and three vertical" urbanization strategy. It has the largest train marshalling station in Asia, the largest zero-load freight transfer station in China and the "meter" high-speed rail network. It is an important comprehensive transportation hub in China.

In addition, Zhengzhou is one of China's commodity distribution centers and one of the main components of China (Henan) Free Trade Experimental Zone; Zhengzhou Airport is China's first national airport economic comprehensive experimental zone; Convenient integrated transportation hubs and large-scale commodity distribution centers have become the great advantages of the development of multimodal transport in Zhengzhou.

(1) Public railway transport

In February 2016, China Railway Corporation formulated the "13th Five-Year Development Plan for Railway Cold Chain Network Layout" and planned "7 cold chain main channels, 13 secondary channels, and 82 cold chain logistics bases”. It has formed the layout structure of China's railway cold chain network, and put forward the development goal of "forming a railway cold chain logistics network with reasonable layout and perfect functions”.

Highway short-haul transportation is flexible and can realize "door-to-door" transportation, but the transportation volume is small and the long-distance transportation cost is large. Railway cold chain logistics has a unique advantage in the medium and long distance. In medium and longdistance transportation, the railway has high speed, large capacity, low energy consumption and less impact by the weather. At the same time, the railway adopts the "BX1K reefer container special flat car + refrigerated storage box"mode for transportation, which can realize the whole transportation refrigeration, guarantee the transportation quality of cold chain goods and bring important guarantee to the normal life of residents. In addition, the railway has the advantage of inter-provincial emergency allocation speed, which can provide a safe, high-quality, fast, economic, all-weather transport platform.

In this regard, Zhengzhou fresh cold chain logistics should rely on the province's large multimodal transport enterprises, vigorously develop the public rail transport, and improve the distribution efficiency and cold chain logistics service level by means of the railway network extending in all directions and flexible and convenient road transport.

(2) Land and air transport

According to the Zhengzhou Daily, the value of cross-border import and export of Zhengzhou Airport Port in 2017 was 1.453 billion yuan, and aviation logistics grew by the trend. Air transportation is a relatively fast and time-saving mode of transportation. It is reported that the second phase of Zhengzhou Airport has been completed and put into operation, with an annual handling capacity of 700,000 tons of cargo. Zhengzhou Airport has opened 34 global freighter routes. It is the fourth largest cargo airport in mainland China and has a good air cargo base.

In addition, Zhengzhou is an international modern integrated transportation hub. The "threenetwork integration" and "four-port linkage" collection and distribution system provide a strong support for the construction of Zhengzhou-Luxembourg "Aerial Silk Road”.

Zhengzhou should combine the background of the Belt and Road, build the ZhengzhouLuxembourg “Aerial Silk Road”, and innovate the development of land and air transport for pilot operation. 


\subsubsection{Adapt to the new retail trend}

New retailing is a new commercial format formed by reconstructing modern commercial elements such as "people, goods and markets" with large data and the Internet.

Jack Ma first put forward the concept of "new retail" in October 2016. This model makes consumer demand information in the supply chain more smoothly. Enterprises can not only provide customers with personalized and customized products and services, but also improve operational flexibility. At the same time, in the whole chain, inventory data of each link is opened and shared in real time. Information flow is closely integrated with logistics.

Zhengzhou cold chain logistics should conform to the trend of new retail and combine with the construction of online Silk Road and the development of cross-border fresh logistics. Through the integration of resources and information sharing, we should plan transportation and distribution reasonably to improve the efficiency of cold chain logistics, reduce logistics costs and improve customer satisfaction with cold chain logistics experience.

\subsubsection{The introduction of new energy and professional talents}

Energy consumption and pollution have always been a prominent problem in the logistics industry. The Ministry of Transportation has formulated a three-year plan of action (2018-2020) for the transportation service to win the victory of building a well-off society in an all-round way and to start the new journey of building a socialist modernization country in an all-round way. It has made a comprehensive deployment of the transportation work in the next three years after the 13th FiveYear Plan. In order to prevent and control air pollution, the Ministry of Transport will promote the upgrading of vehicle structure, promote the use of new energy vehicles, and vigorously eliminate old vehicles. In this regard, cold chain logistics enterprises should adopt new energy or clean energy vehicles instead of vehicles with serious tail gas pollution. This not only reduces the operation cost of enterprises, but also greatly reduces the social cost.

The lack of professional personnel is also a key factor restricting the development of cold chain logistics. Enterprises should introduce professional cold chain management personnel, train and reeducate employees, standardize enterprise operation and management, bid farewell to empirical management mode, improve service quality, and shape the core competitiveness of enterprises.

\subsubsection{Strengthen government supervision and policy support}

Optimize the traffic management of freight vehicles. It will be convenient for all enterprises to obtain certificates and pass the whole line, and to engage in the distribution of fresh agricultural products and refrigerated fresh-keeping products and the use of energy-saving and new-energy vehicles.

Broaden the investment and financing channels of logistics enterprises (Fugui Zheng,2017). Support the development of innovative logistics companies. Strengthen logistics development planning and land use support, and provide convenience and preferential policies for logistics enterprises. Support the construction of local third-party cold chain logistics enterprises, strengthen the overall planning of cold chain logistics infrastructure equipment, encourage the construction of cold chain facilities and equipment in the initial processing of production areas, strengthen the construction of urban distribution infrastructure equipment, and strive to solve the "first mile" and " The last mile" question.

Implement and improve taxation policies in the logistics sector to reduce and standardize logistics charges. Implement the "green" channel policy for fresh agricultural products transportation, focus on solving problems such as "disorderly charges and fines", reduce the operating costs of logistics enterprises, standardize the competition and operation of Zhengzhou 
fresh cold chain logistics market, and create a good industrial environment for the logistics industry.

\section{Conclusions}

The seasonality and perishability of fresh products have strict requirements for cold chain logistics. Zhengzhou cold chain logistics has a good development foundation, but there are still problems such as backward facilities, low informationization, and high cost of cold chain logistics. Zhengzhou cold chain logistics should combine the background of the times, adapt to the development trend, strengthen the construction of cold chain logistics facilities, improve the information level of cold chain logistics, reduce logistics costs to improve the cold chain logistics service level and customer satisfaction.

\section{References}

[1] Fugui Zheng, 2017.Accelerator of Supply Chain Finance into Cold Chain Logistics, China Logistics and Purchasing. (24), pp.114

[2] Jianqi Zhang, 2018 .Perspective and Countermeasures for the Development Foundation and Situation of Agricultural Products Cold Chain Logistics, Logistics Engineering and Management. 40 (02), pp.7-11.

[3] Qinggang Zhao, 2011.Research on the Status Quo and Countermeasures of China's Cold Chain Logistics Development, China Circulation Economy.25(02),pp.24-28.

[4] Xueguo Yuan, Ping Zou, Jun Zhu, Di Wu, 2015.The development trend, problems and countermeasures of China's cold chain logistics industry.17 (01), pp.7-14.

[5] Yafang Tian , Xu Feng,2017. Analysis on the Integration of Intelligent Warehouse Management System and Enterprise Information System, Internet of Things Technology. 7(06), pp. 71-72+76. 